ISSN 0103-9954

\title{
AVALIAÇÃO DA RESISTÊNCIA AO CISALHAMENTO E À DELAMINAÇÃO EM MADEIRA LAMINADA COLADA
}

\author{
EVALUATION OF STRENGTH TO SHEAR AND DELAMINATION IN GLUED LAMINATED \\ WOOD
}

\author{
Carlito Calil Neto ${ }^{1}$ André Luis Christoforo ${ }^{2}$ Sérgio Luiz Moni Ribeiro Filho ${ }^{3}$ \\ Francisco Antonio Rocco Lahr ${ }^{4}$ Carlito Calil Junior ${ }^{5}$
}

\begin{abstract}
RESUMO
A Madeira Laminada Colada (MLC) apresenta uma grande gama de aplicações. No Brasil, o seu emprego na forma de cruzetas para postes de rede aérea de distribuição de energia elétrica tem despertado a atenção de companhias do ramo, motivadas pelo potencial de emprego deste material. Dentre os fatores que influenciam o desempenho mecânico de soluções em MLC destacam-se a eficiência e a afinidade dos adesivos para com as espécies de madeiras utilizadas, o tipo de tratamento e o teor de umidade das lâminas de madeira, motivando o desenvolvimento de novas pesquisas nesta temática. Este trabalho objetivou investigar, via Método de Planejamento Fatorial de Experimentos (DOE), a influência dos fatores tipo de madeira (pinus; teca; eucalipto), adesivo (Purbond; Cascophen) e tratamento (CCA; CCB; CCBS) nas variáveis respostas resistência ao cisalhamento e à delaminação, consistindo nos mesmos fatores e combinação avaliados no projeto de Pesquisa e Desenvolvimento da ANEEL/EESC-PD220-07: Cabeça de Série da Cruzeta de MLC. Os resultados da análise estatística revelaram que o fator tipo de madeira expressou efeito significativo para ambas as variáveis respostas avaliadas, o mesmo não ocorrendo com os fatores tipo de adesivo e de tratamento. Já o teor de umidade mostrou ser significativo em todas as espécies de madeira quando analisada a resistência ao cisalhamento, apresentando a madeira de teca a maior resistência ao cisalhamento e à delaminação.
\end{abstract}

Palavras-chave: madeira laminada colada (MLC); planejamento fatorial de experimentos (DOE); cruzetas; delaminação.

\section{ABSTRACT}

The Glued Laminated Wood has a large range of applications. In Brazil, its employment as cross-piece poles for overhead electrical power has attracted the attention of companies in the industry, motivated by the potential use of this material. Among the factors that influence the mechanical performance of Glulam solutions stand out efficiency and affinity of the adhesives to the species of wood used, the type of treatment and moisture content of wood veneer, motivating the development of new research on this topic. This research aimed to investigate, by Design of Experiments (DOE), the influence of wood (pinus, teca,

1 Engenheiro Industrial Madeireiro, Msc., Departamento de Engenharia Civil, Escola de Engenharia de São Carlos, Universidade de São Paulo, Av. Trabalhador Sãocarlense, 400, Bairro Centro, CEP 13560-970, São Carlos (SP), Brasil. netousp@gmail.com

2 Engenheiro Civil, Dr., Departamento de Engenharia Mecânica, Universidade Federal de São João del-Rei, Praça Frei Orlando, 170, Bairro Centro, CEP 36307-352, São João del Rei (MG), Brasil. alchristoforo@yahoo.com.br

3 Engenheiro Mecânico, Departamento de Engenharia Mecânica, Universidade Federal de São João del-Rei, Praça Frei Orlando, 170, Bairro Centro, CEP 36307-352, São João del Rei (MG), Brasil. sergiolmrf@gmail.com

4 Engenheiro Civil, Dr., Departamento de Engenharia de Estruturas, Escola de Engenharia de São Carlos, Universidade de São Paulo, Av. Trabalhador Sãocarlense, 400, Bairro Centro, CEP 13560-970, São Carlos (SP), Brasil. frocco@sc.usp.br

5 Engenheiro Civil, Dr., Departamento de Engenharia De Estruturas, Escola de Engenharia de São Carlos, Universidade de São Paulo, Av. Trabalhador Sãocarlense, 400, Bairro Centro, CEP 13560-970, São Carlos (SP), Brasil. calil@sc.usp.br 
eucalipto), adhesive (Purbond; Cascophen) and treatment (CCA, CCB, CCBS ) in the variable responses shear strength and delamination, consisting in the same combination factors evaluated in ANEEL/EESCPD220-07 project: Head Crosshead Glulam Series. The results of the statistical analysis showed that the species factor expressed significant effect for both response variables evaluated, did not occur with adhesive and treatment factors. Moisture content was significant in the wood evaluated when analyzed the shear strength, and the teca wood showed the highest shear strength and also relating to the delamination.

Keywords: glued laminated wood; design of experiments (DOE); crosshead; delamination.

\section{INTRODUÇÃO}

Dentre os principais materiais utilizados na confecção de estruturas destaca-se a madeira, por ser um material de fonte renovável e de excelente relação resistência/densidade, chegando a ser quatro vezes superior a do aço (CALIL et al., 2003).

Nos últimos anos, devido à grande preocupação com o impacto ambiental, sustentabilidade e necessidade de utilizar elementos estruturais de qualidade, juntamente com o avanço na tecnologia dos adesivos, ocorreram também grandes avanços nos produtos derivados da madeira, principalmente pela possibilidade do emprego de madeiras provenientes de florestas plantadas, tornando esses produtos ecologicamente corretos, propiciando assim novos campos de aplicação e garantindo um sólido mercado consumidor. Além da redução dos agravos ambientais, os produtos engenheirados de madeira proporcionam um menor uso da madeira maciça e praticamente a utilização de $100 \%$ de matéria-prima para sua manufatura (CALIL NETO, 2011).

Dentre as soluções alternativas ao uso da madeira maciça destaca-se a Madeira Laminada Colada (MLC), podendo ser empregada sob as mais variadas formas estruturais, consistindo em um produto que requer precisão de fabricação em todos os seus estágios.

Com relação à produção de MLC, alguns fatores tais como: espécies de madeira utilizadas, espessuras e posições das lâminas, tipo de adesivo e tratamentos, entre outras, podem afetar significativamente a resistência mecânica para o qual as peças foram projetadas (CALIL, 2010), motivando o estudo e a elaboração de uma série de pesquisas, podendo-se citar as desenvolvidas por Graeff (1995), Macedo (2000), Zangiácomo (2003) e Fiorelli (2005), sendo estudados: a eficiência do emprego de emendas de topo, a fadiga em emendas dentadas, o emprego de espécies tropicais na elaboração de vigas de MLC e o uso de fibras de vidro como reforço em vigas de MLC respectivamente.
O adesivo se apresenta como uma das variáveis mais influentes no desempenho mecânico. A seleção do adesivo utilizado para elementos estruturais de madeira depende fundamentalmente das condições do ambiente onde este se insere e das condições de exposição (PIZZI, 1994).

Os adesivos comumente utilizados na fabricação de madeira laminada colada no Brasil são os poliuretanos e os à base de resorcinol, com cura à temperatura ambiente. Tais adesivos apresentam alta resistência à umidade, tornandoos aptos para uso exterior. Entretanto, a madeira utilizada neste tipo de ambiente requer um processo de impregnação preservativa à demanda biológica. O principal problema é que os adesivos comerciais nem sempre aderem à madeira tratada de forma consistente, de maneira a satisfazer as exigências industriais referentes à resistência à delaminação (VICK et al., 1996).

Não só os fatores, mas também a afinidade da interação entre ambos podem acarretar em diferenças significativas no desempenho de estruturas elaboradas em MLC. Neste âmbito, Zhang e Kamdem (2000) apontam que o tratamento por CCA pode comprometer a eficiência da adesão entre lâminas, pois os materiais metálicos insolúveis contidos no CCA precipitam sobre a parede celular da madeira, interferindo no contato madeira-resina, reduzindo a durabilidade da união interfacial.

Como a utilização de produtos de MLC ainda não é muito difundida no Brasil é evidente que estudos devem ser realizados na investigação dos fatores espécies de madeira, adesivo e tratamento para uma caracterização das madeiras que melhor se adaptem a essa combinação (CALIL NETO, 2011), devendo ter destaque, nessas pesquisas, principalmente as madeiras provenientes de florestas plantadas.

Este trabalho objetiva investigar, com o auxílio do método de planejamento fatorial de experimentos, a influência das composições entre tipos de madeira, tipos de adesivo, teores de umidade e formas de tratamentos preservativos da madeira 
na resistência ao cisalhamento e à delaminação em corpos de prova extraídos de MLC, consistindo nos mesmos fatores avaliados no projeto de Pesquisa e Desenvolvimento da ANEEL/EESC-PD220-07: Cabeça de Série da Cruzeta de MLC, permitindo gerar subsídios para a utilização da madeira de reflorestamento tratada e sem tratamento em MLC.

\section{MATERIAL E MÉTODOS}

A Tabela 1 explicita os níveis associados aos fatores experimentais investigados neste trabalho.

Os quatro fatores de entrada apresentados na Tabela 1 são utilizados para avaliar a resistência ao cisalhamento, enquanto que para a resistência à delaminação são utilizados apenas três: tipo de madeira, tipo de adesivo e tipo de tratamento. O ensaio de resistência ao cisalhamento na linha de cola e na madeira sólida nas condições seca e saturada para as das vigas em MLC foram efetuados no Laboratório de Madeiras e de Estruturas de Madeira (LaMEM), Departamento de Engenharia de Estruturas, Escola de Engenharia de São Carlos.

Os adesivos utilizados neste trabalho são o Cascophen RS-216M e o Purbond HBS309. O Cascophen RS-216M é um produto formulado por uma resina à base de resorcinolformol e um Endurecedor (FM60M). Os dois componentes depois de misturados resultam em um adesivo de alto desempenho, à prova d'água (fria ou fervente) e resistente à ação de fungos e intempéries. $O$ Purbond é adesivo monocomponente (sem mistura), contendo $100 \%$ sólidos (não contém solvente), requerendo um tempo de aplicação de 30 minutos e tempo de cura de 75 minutos.

A gramatura da cola especificada pelo fabricante (linha simples) é de 180 a $200 \mathrm{~g} / \mathrm{m}^{2}$ a uma pressão de $10 \mathrm{MPa}$, entretanto, a gramatura utilizada para os dois adesivos na fabricação foi de 300 a $350 \mathrm{~g} / \mathrm{m}^{2}$, ocasionadas pelo uso de pincéis na sua dispersão. A Figura 1a ilustra o espalhamento manual do adesivo entre as lamelas de madeira e a Figura $1 \mathrm{~b}$ o processo de prensagem utilizado para formação da MLC.

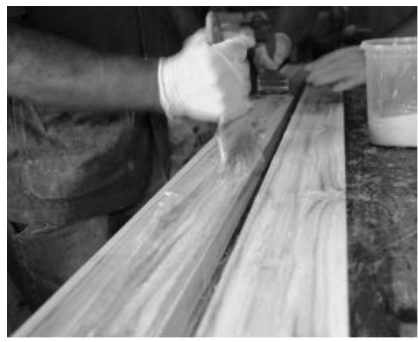

(a)

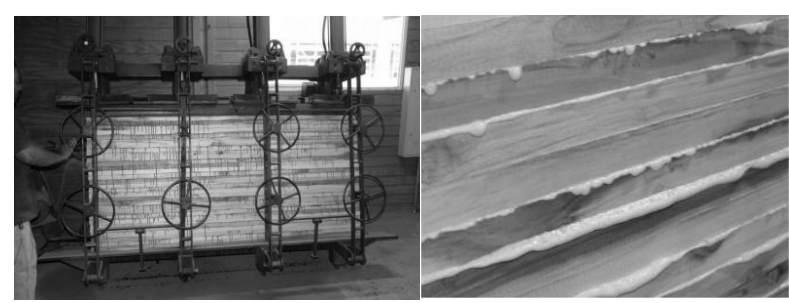

(b)

FIGURA 1: Aplicação do adesivo nas lamelas e montagem das MLC.

FIGURE 1: Adhesive application and assembly of MLC.

Após a montagem, foram separadas 54 cruzetas de madeiras de reflorestamento, de dimensões $4 \times 10 \times 210 \mathrm{~cm}$, referentes ao projeto de pesquisa e desenvolvimento da ANEEL/EESC, para que fossem tratadas com os preservativos à base de CCA, CCB e CCBS. Os tratamentos químicos foram realizados em autoclave após sete dias de cura do adesivo, nas dependências da empresa Prema Tecnologia e Comércio S. A. (Rio Claro-SP) e na I. M. Dib \& Cia Ltda. (Sengés - PR). Em seguida, os corpos de prova foram climatizados até atingirem teor de umidade $12 \%$. As madeiras eucalipto, pinus e teca utilizadas foram fornecidas pela Aracruz (RS), Agrotec Empreendimentos Agropecuários Ltda.

TABELA 1: Fatores de entrada e níveis.

TABLE 1: Input factors and levels.

\begin{tabular}{ccccc}
\hline Fatores de Entrada & \multicolumn{4}{c}{ Níveis } \\
\hline Tipo de madeira & pinus & teca & eucalipto & - \\
Adesivo & Purbond & Cascophen & - & - \\
Tratamento & sem & CCA & CCB & CCBS \\
Teor de umidade & $12 \%$ & saturada & - & - \\
\hline
\end{tabular}




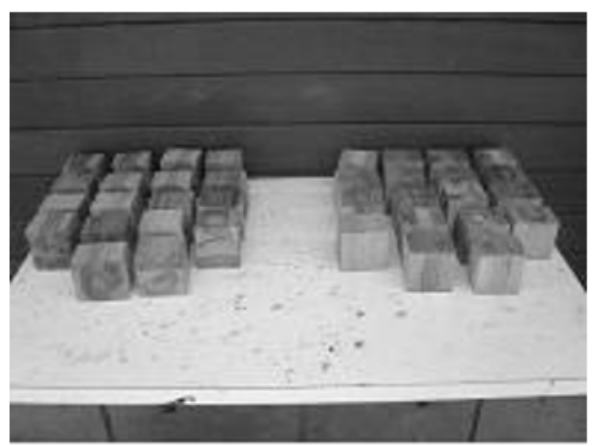

(a)

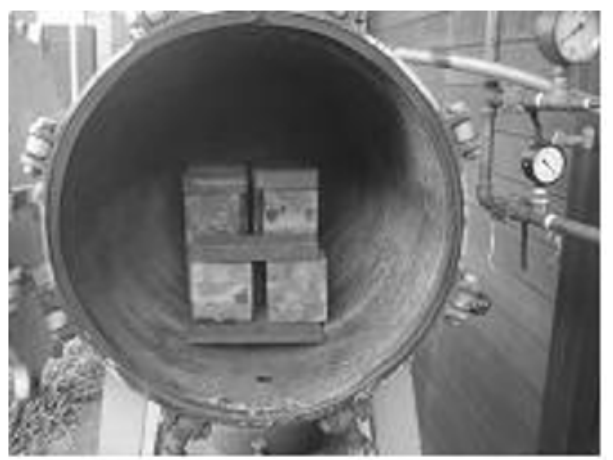

(b)

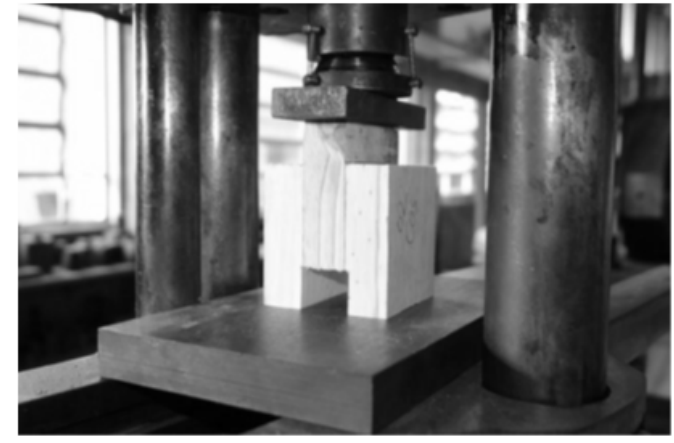

(a)

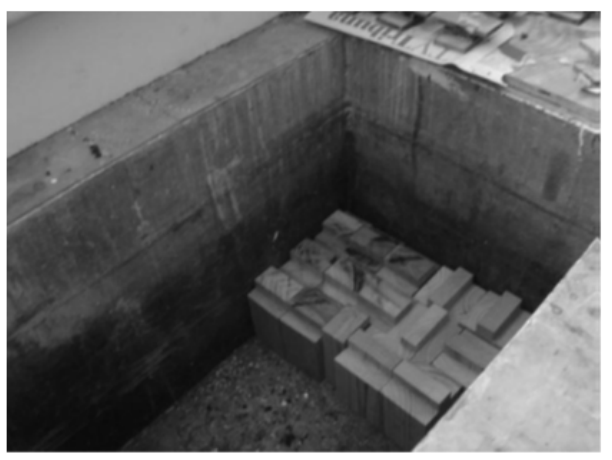

(b)

FIGURA 2: Corpos de prova para ensaios de FIGURA 3: (a) Corpo de prova para o cisalhadelaminação (a) e autoclave (b).

FIGURE 2: Specimens for delamination tests (a) and autoclave (b). mento, (b) corpos de prova no tanque de imersão.

FIGURE 3: (a) Specimens for shear tests, (b) specimens in tank of immersion.

TABELA 3: Resultados da ANOVA para a média das variáveis respostas resistência ao cisalhamento e à delaminação.

TABLE 3: ANOVA results for the average of shear and delamination variables.

\begin{tabular}{cccc}
\hline & Fatores Experimentais & Cisalhamento & Delaminação \\
\hline Madeira & 0,000 & 0,004 \\
& Adesivo & 0,517 & 0,924 \\
& Tratamento & 0,878 & 0,228 \\
& Umidade & 0,000 & - \\
\hline & Madeira - Adesivo & 0,155 & 0,866 \\
& Madeira - Tratamento & 0,975 & 0,000 \\
& Madeira - Umidade & 0,422 & - \\
& Adesivo - Tratamento & 0,548 & 0,993 \\
& Adesivo - Umidade & 0,710 & - \\
\hline & Tratamento - Umidade & 0,933 & - \\
\hline
\end{tabular}


(GO) e Indústria Floresteca (MT), respectivamente. De cada cruzeta foram retirados cinco corpos de prova, sendo dois para os testes de resistência ao cisalhamento e três para os testes de delaminação. Os testes de resistência ao cisalhamento foram desenvolvidos segundo as premissas da norma brasileira ABNT NBR 7190:1997 (Projeto de Estruturas de Madeira), enquanto que os referentes à delaminação foram executados de acordo com a norma canadense CSA 0177:2006 (Qualification Code for Manufactures of Structural Glued Laminated Timber).

Após o corte, os corpos de prova para os ensaios de delaminação (Figura 2a) foram encaminhados para a autoclave (Figura 2b), iniciando-se o ciclo de delaminação.

Para os ensaios de cisalhamento (Figura 3a), de cada condição experimental foi preparado um corpo de prova submerso em tanque (Figura 3b) com água fria por 48 horas.

\section{RESULTADOS E DISCUSSÃO}

Os planejamentos fatoriais foram realizados na identificação dos efeitos principais (significativos) e interações dos fatores sobre as variáveis investigadas (MONTGOMERY, 2005). A Tabela 3 apresenta os resultados da ANOVA (P-valor) para a média das respostas resistência ao cisalhamento e à delaminação.

Os fatores relacionados a $\mathrm{P}$-valor menor ou igual a 0,05 (95\% de confiabilidade) são considerados significativos (WERKEMA e AGUIAR, 1996), estando sublinhados na Tabela 3. As Figuras 4a e 4b

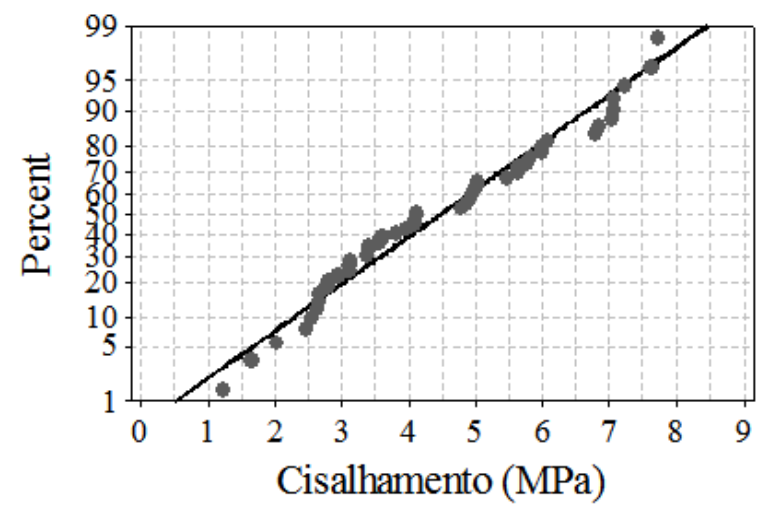

(a) exibem os gráficos de probabilidade normal para as variáveis respostas resistência ao cisalhamento e à delaminação.

$\mathrm{Na}$ Figura 4a, os pontos distribuídos uniformemente ao longo da reta atendem às condições de normalidade exigidas para validação do modelo da ANOVA (CHOU et al., 1998; ROWLANDS E ANTONY, 2003), o mesmo não ocorreu com a delaminação (Figura 4b), sendo utilizada a transformação de Johnson para a normalização e homogeneidade dos dados (Figura 5).

Os valores experimentais da resistência ao cisalhamento variaram entre 1,21 $\mathrm{MPa}$ e 7,73 $\mathrm{MPa}$. $\mathrm{O}$ tipo de madeira e o teor de umidade influenciaram significativamente na resistência ao cisalhamento, exibindo P-valores 0,000 e 0,000 (Tabela 3). A Figura 6 exibe os gráficos de efeitos principais dos tipos de madeira e do teor de umidade em relação à resistência ao cisalhamento.

Em análise dos efeitos significativos, verificou-se uma diferença percentual $44,38 \%$ na resistência ao cisalhamento entre as madeiras de teca e de eucalipto, e de $38,90 \%$ entre as madeiras de pinus e eucalipto (Figura 6a).

O gráfico de efeitos principais do teor de umidade da madeira demonstra sua influência na resistência ao cisalhamento (Figura 6b). Como esperado, a saturação da madeira é inversamente proporcional à resistência ao cisalhamento, apresentando uma diferença percentual de $34,35 \%$ entre as condições com $12 \%$ de teor de umidade e saturada.

A Figura 7 exibe a interação entres os fatores tipo de madeira e teor de umidade da madeira sobre

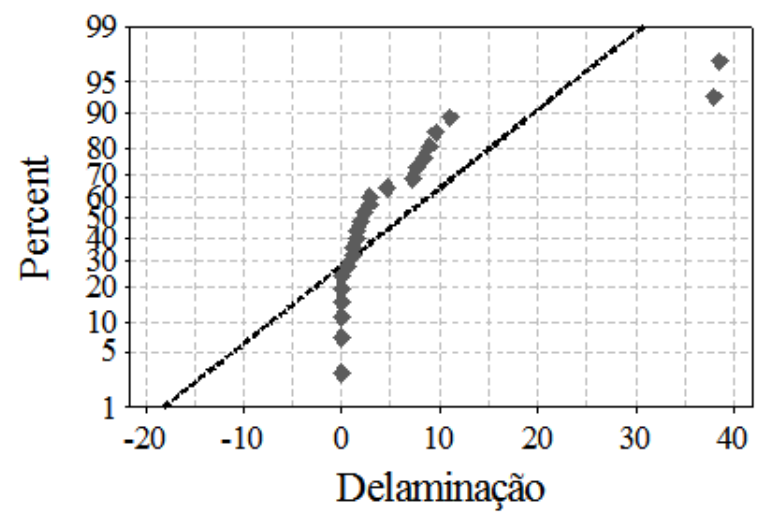

(b)

FIGURA 4: Teste de normalidade das variáveis respostas resistência ao cisalhamento (a) e à delaminação (b).

FIGURE 4: Normality tests of shear strength (a) and delamination (b). 

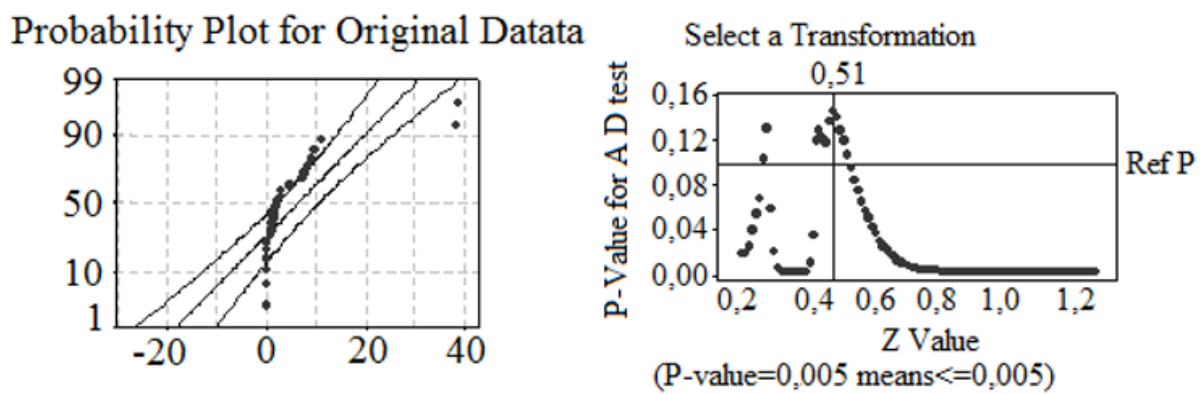

Probability Plot for Transformed Data

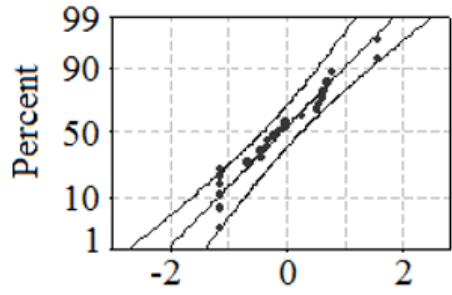

P-Value for Best Fit: 0,17434

$Z$ for Best Fit: 0,51

Best Transformation Type pe: SL

Transformation function equals

$-0,819828+0,644233 * \log (X+0,607262)$

FIGURA 5: Transformação de Johnson para normalização dos dados referentes à delaminação.

FIGURE 5: Johnson's transformation for the normalization of data related to delamination.

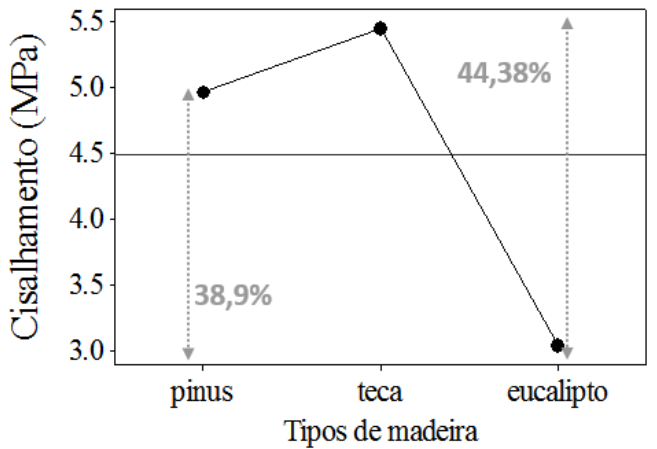

(a)

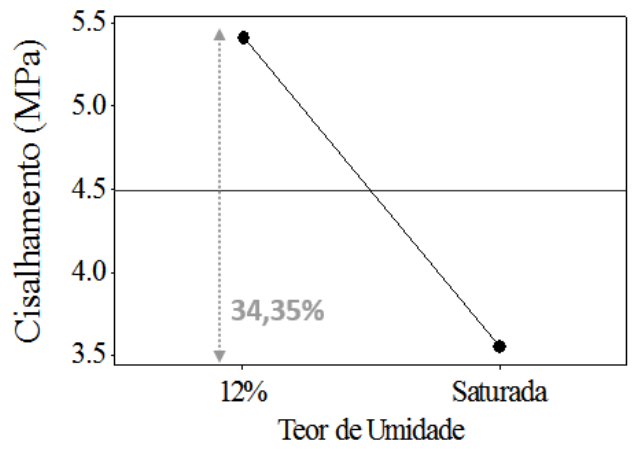

(b)

FIGURA 6: Efeito da madeira (a) e do teor de umidade (b) sobre a resistência ao cisalhamento (MPa). FIGURE 6: Species effect (a) and moisture content (b) on shear strength (MPa).

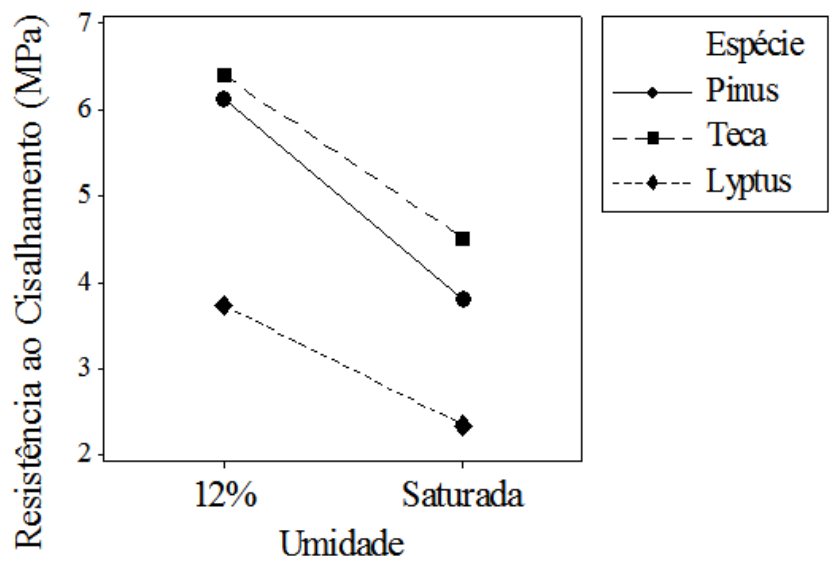

FIGURA 7: Interação entre os fatores espécie e umidade sobre a resistência ao cisalhamento (MPa). FIGURE 7: Specie and moisture content factors interaction on shear strength (MPa). 
a variável resposta resistência ao cisalhamento. Verifica-se que a melhor combinação experimental foi obtida da madeira de teca com teor de umidade de $12 \%$.

Os valores da delaminação nas madeiras variam entre $0 \%$ e 38,6\%. Através da análise de variância pode-se observar, com $95 \%$ de significância, que o fator de entrada tipo de madeira e a interação entre o tipo de tratamento e tipo de madeira (Tabela 3) influenciaram de forma significativa na delaminação. Em análise dos efeitos e interações observou-se que a madeira de eucalipto e a condição experimental sem tratamento (ST) foram significativas (Figura 8), ocorrendo nesta condição a maior delaminação.

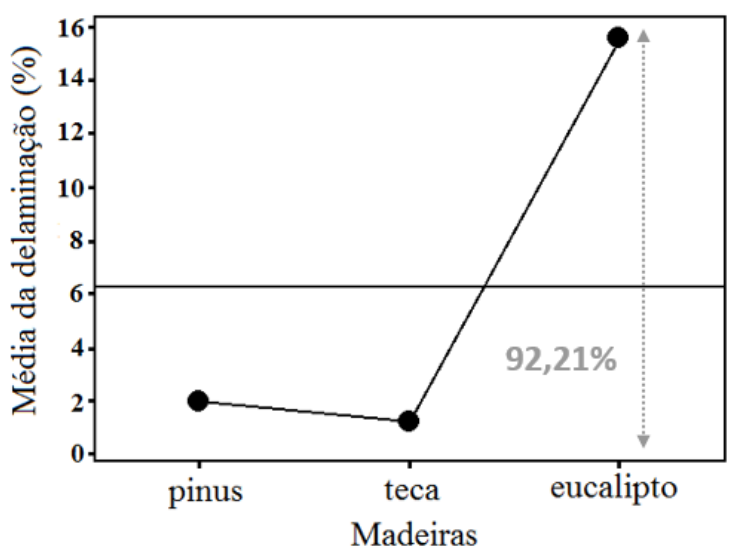

(a)

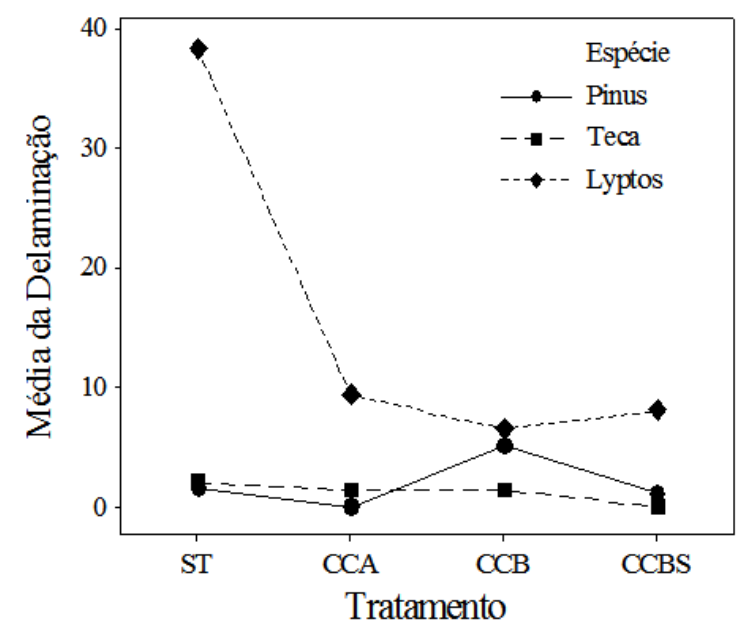

(b)

FIGURA 8: Efeito do fator Espécie (a) e interação Espécie-Tratamento sobre a delaminação (b).

FIGURE 8: Species factor (a) and interaction species-treatment on delamination response (b).

\section{CONCLUSÕES}

O planejamento fatorial foi empregado para investigar a influência dos fatores: tipo de madeira, tipo de adesivo, teor de umidade e tipo de tratamento preservativo na resistência ao cisalhamento e à delaminação em corpos de prova provenientes de cruzetas em MLC referentes ao projeto de Pesquisa e Desenvolvimento da ANEEL/EESC-PD220-07.

Os resultados experimentais revelaram que o fator tipo de madeira expressou efeito significativo para ambas as propriedades avaliadas, apresentando a madeira de teca a maior resistência ao cisalhamento, sendo apenas $9,90 \%$ superior à resistência ao cisalhamento da madeira de pinus, e maior porcentagem de delaminação para a madeira de eucalipto.

Os fatores tipo de adesivo e tipo de tratamento preservativo não foram influentes nas variáveis respostas investigadas, exibindo P-valores acima de 0,05 .

Diante das condições experimentais realizadas, a madeira de teca apresentou-se como a melhor opção dentre as madeiras utilizadas nas experimentações, por conferir maior resistência ao cisalhamento e por exibir a menor porcentagem de delaminação, podendo ser utilizada com qualquer tipo de adesivo ou tratamento aqui investigado, assim como a madeira de pinus, apresentando comportamento semelhante ao da madeira teca. A madeira de eucalipto não foi adequada para caracterização do processo Madeira Laminada Colada (MLC), uma vez que apresentou baixa resistência ao cisalhamento e alta porcentagem de delaminação.

\section{REFERÊNCIAS BIBLIOGRÁFICAS}

ABNT. Projeto de Estruturas de Madeira. NBR 7190, Rio de Janeiro, 1997.

CALIL NETO, C. Madeira laminada colada (MLC): controle de qualidade em combinações espécie-adesivo-tratamento-preservativo. São Carlos, 2011. Dissertação de Mestrado - Escola de Engenharia de São Carlos da Universidade de São Paulo, São Carlos, 2011.

CALIL, C. JR.; LAHR, F. A. R.; DIAS, A. A. Dimensionamento de elementos estruturais de madeira. Barueri: Manole, 2003.

CALIL, C. N. A Madeira Laminada Colada. Revista da Madeira, Caxias do Sul, 5 p. Artigo técnico. Disponível em http://www.remade.com.br, 2010. 
CANADIAN STANDARDS ASSOCIATION - CSA 0177:2006. Qualification Code for Manufactures of Structural Glued. Laminated Timber. 16 p.

CHOU, Y.; POLANSKY A. M.; MASON R. L. Transforming non-normal data to normality in statistical process control. Journal of Quality Technology, p. 133-141, Apr. 1998.

FIORELLI, J. Estudo teórico e experimental de vigas de madeira laminada colada reforçadas com fibras de vidro. 2005. Tese de Doutorado Escola de Engenharia de São Carlos da Universidade de São Paulo, São Carlos, 2005.

GRAEFF, A. Estudo da madeira laminada colada com emendas de topo reforçadas com fibras de vidro. 1995. Tese de Doutorado - Universidade Federal de Santa Catarina, Florianópolis, 1995.

MACEDO, A. N. Fadiga em emendas dentadas em madeira laminada colada. 2000. Tese de Doutorado - Escola de Engenharia de São Carlos da Universidade de São Paulo, São Carlos, 2000.

MONTGOMERY, D. C. Design and analysis of experiments. 6th ed.. Arizona: John Wiley \& Sons Inc., 2005.
PIZZI, A. Advanced wood adhesives technology. New York: Marcel Dekker, 1994. 297 p.

ROWLANDS, H.; ANTONY, F. Application of design of experiments to a spot welding process. Assembly Automation, v. 23, n. 3, p. 273-279, 2003. VICK, C. B.; GEIMER, R. L.; WOOD, J. E. Flakeoards from recycled CCA-treated southern pine lumber. Forest Products Journal, v. 46, p.89-91, 1996.

WERKEMA, M. C. C.; AGUIAR, S. Planejamento e análise de experimentos: Como identificar e avaliar as principais variáveis influentes em um processo. Belo Horizonte: Fundação Christiano Ottoni, Escola de Engenharia da UFMG, 1996.

ZANGIÁCOMO, A. L. Emprego de espécies tropicais alternativas na produção de elementos estruturais de madeira laminada colada. 2003. Dissertação de Mestrado - Escola de Engenharia de São Carlos da Universidade de São Paulo, São Carlos, 2003.

ZHANG, J.; KAMDEM, D. P. Interaction of copperamine with southern pine. Wood Fiber Science, v. 32, p. 332-339, 2000. 